\title{
On the correspondence between two- and three-dimensional Eshelby tensors
}

\author{
In occasion of the 80th birthday of Prof. Wojciech Pietraszkiewicz
}

Received: 25 January 2019 / Accepted: 19 February 2019 / Published online: 6 March 2019

(C) The Author(s) 2019

\begin{abstract}
We consider both three-dimensional (3D) and two-dimensional (2D) Eshelby tensors known also as energy-momentum tensors or chemical potential tensors, which are introduced within the nonlinear elasticity and the resultant nonlinear shell theory, respectively. We demonstrate that 2D Eshelby tensor is introduced earlier directly using 2D constitutive equations of nonlinear shells and can be derived also using the throughthe-thickness procedure applied to a 3D shell-like body.
\end{abstract}

Keywords Eshelby tensor $\cdot$ Nonlinear elasticity $\cdot$ Nonlinear shell $\cdot$ Phase transformations $\cdot$ Through-thethickness integration

\section{Introduction}

Eshelby tensor known also as the Eshelby stress tensor or the energy-momentum tensor or the tensor of chemical potential was introduced originally in pioneering works by Eshelby [27-29]. Since it describes the energy release rate for a moving singularity, the Eshelby tensor found various applications in the theory of fracture $[39,41,47,48]$, in the theory of stress-induced phase transitions [2,9,11,38,49], and in the modelling of the stress-assisted chemical reaction fronts propagation [32,34,58]. In particular, the properties of the Eshelby tensor result in several path-independent line integrals, see, e.g. [41,47,48]. For modelling of stress-induced phase transformations, the jump of discontinuity of the Eshelby tensor across the phase interface is used for the formulation of the thermodynamic compatibility condition which is necessary for determination of the interface position, see $[2,9,33,38,64]$. Motivating by the behaviour of martensitic films $[12,13,30,44,50]$ and biological membranes [3,15,53], Eremeyev and Pietraszkiewicz [24] proposed the 2D model of thin-walled structures undergoing phase transitions within the nonlinear shell theory presented in [16,45,46,55]. In [24], the thermodynamic compatibility condition on the phase interface was derived using the stationarity of the total energy functional expressed through 2D constitutive equations. This description was further extended for more complex cases such as quasistatic deformations [26], viscoelastic properties [25], and the influence of a line tension [56]. The latter case constitutes an example of singular curves in shells discussed in [57] in detail. The analysis performed in $[24,26]$ demonstrated that the developed $2 \mathrm{D}$ model can capture essential peculiarities of deformations of thin films made of shape memory alloys observed experimentally, see, e.g. $[12,13,30,44,50]$.

Communicated by Holm Altenbach.

V. A. Eremeyev · V. Konopińska-Zmysłowska $(\bowtie)$

Gdańsk University of Technology, ul. G. Narutowicza 11/12, 80-233 Gdańsk, Poland

E-mail: violetta.konopinska@pg.edu.pl

V. A. Eremeyev

E-mail: eremeyev.victor@gmail.com 


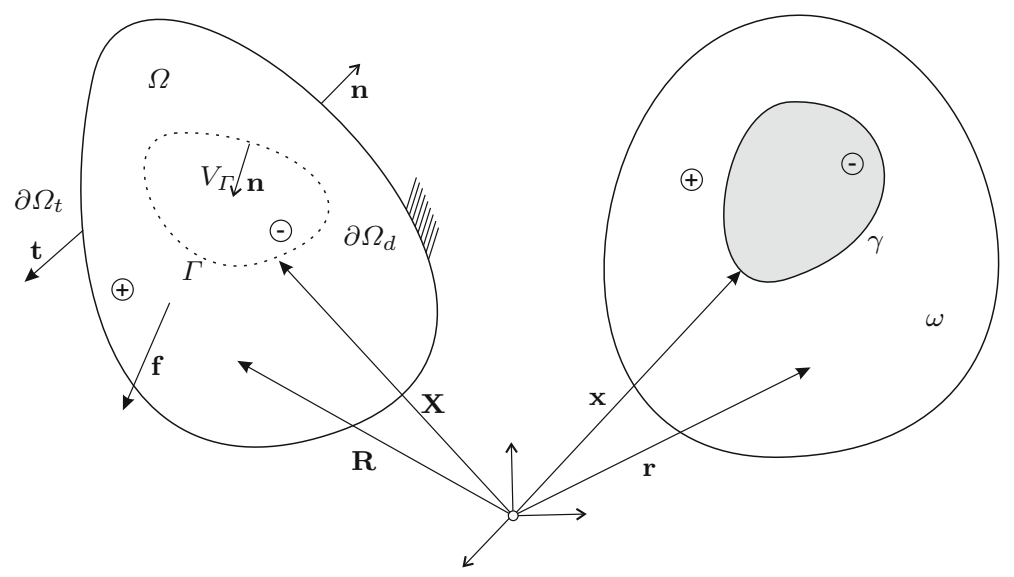

Fig. 1 Deformation of a 3D solid undergoing phase transition

The aim of this paper is to establish straightforward correspondence between 2D Eshelby tensor and its 3D counterpart. In other words, we are looking for a dependence which is similar to the dependencies between the first Piola-Kirchhoff stress tensor and the stress resultant and moment tensors in the resultant theory of shells $[16,45,46]$.

The paper is organized as follows. In Sect. 2, we briefly recall the basic relations of equilibrium of twophase solids followed from the stationarity of the total energy functional $[8,38]$. Following [24] in Sect. 3, we introduce 2D Eshelby tensor and other 2D fields used in the nonlinear resultant shell theory. Finally, in Sect. 4 we consider a shell-like solid undergoing phase transformation. In order to find a dependence between 2D and 3D Eshelby tensors, we perform the through-the-thickness integration along the thickness coordinate of the thermodynamic compatibility conditions on the phase interface.

\section{Eshelby tensor and equilibrium conditions of 3D solids undergoing phase transition}

Let us consider a three-dimensional solid which occupies in reference and current placement volumes $\Omega$ and $\omega$, respectively. The deformation of an elastic solid can be described through the one-to-one mapping $\Omega \rightarrow \omega$

$$
\mathbf{r}=\mathbf{r}(\mathbf{R})
$$

where $\mathbf{r}$ and $\mathbf{R}$ are the position vectors in the current and reference placements, respectively. We assume that in the current placement the solid undergoes martensitic-type phase transitions, so it consists of two phases separated by phase interface $\gamma$. Note that $\gamma$ is a non-material surface, in general. This means that $\gamma$ propagates across material particles. So for two-phase solids in addition to $\mathbf{r}$, we introduce vector $\mathbf{x}$ which specifies the position of $\gamma$. Vector $\mathbf{x}$ plays a role of additional kinematical descriptor responsible for degrees of freedom related to the phase transition. Within the Lagrangian description instead of $\mathbf{x}$, we introduce vector $\mathbf{X}$ such that $\mathbf{r}(\mathbf{X})=\mathbf{x}$, so the image of $\gamma$ under inverse mapping (1) is $\Gamma: \mathbf{r}(\Gamma)=\gamma$, as shown in Fig. 1. Note that $\Gamma$ is also non-material surface defined in the reference placement. In what follows, we denote phases with indices "+" and "-".

Within the variational approach, we consider the stationarity of the total energy functional

$$
\mathcal{E}[\mathbf{r}, \mathbf{X}]=\int_{\Omega}(W-\rho \mathbf{f} \cdot \mathbf{r}) \mathrm{d} \Omega-\int_{\partial \Omega_{\mathrm{t}}} \mathbf{t} \cdot \mathbf{r} \mathrm{d} A
$$

on kinematically admissible fields $\mathbf{r}$ and $\mathbf{X}$, that is when $\delta \mathbf{r}=\mathbf{0}$ at $\partial \Omega_{\mathrm{d}}$. Here, $W$ is the strain energy function, $W=W(\mathbf{F}), \mathbf{F}=\operatorname{Grad} \mathbf{r}$ is the deformation gradient, $\rho$ is the referential mass density, $\mathbf{f}$ and $\mathbf{t}$ are the vectors of mass and surface forces, respectively, $\partial \Omega_{\mathrm{t}}$ is a part of the whole boundary $\partial \Omega$ on which surface forces $\mathbf{t}$ are given. On the rest part, $\partial \Omega_{\mathrm{d}}=\partial \Omega \backslash \partial \Omega_{\mathrm{t}}$ displacements are given: $\mathbf{r}=\mathbf{r}_{0}$. Hereinafter, we define the Lagrangian 
divergence and gradient operators, Div and Grad, as in [62]. For example, in Cartesian coordinates $X_{\mathrm{k}}$ with corresponding unit orthogonal base vectors $\mathbf{i}_{\mathrm{k}}, k=1,2,3$, Grad and Div are calculated by the formulae

$$
\operatorname{Grad}(\ldots)=\frac{\partial}{\partial X_{\mathrm{k}}}(\ldots) \otimes \mathbf{i}_{\mathrm{k}}, \quad \operatorname{Div}(\ldots)=\frac{\partial}{\partial X_{\mathrm{k}}}(\ldots) \cdot \mathbf{i}_{\mathrm{k}},
$$

where " $\otimes$ " and “"” stand for the tensor and scalar products, respectively.

From $\delta \mathcal{E}=0$, we derive the equilibrium equations

$$
\operatorname{Div} \mathbf{P}+\rho \mathbf{f}=\mathbf{0}, \quad \mathbf{R} \in \Omega,
$$

the static boundary conditions

$$
\mathbf{P n}=\mathbf{t}, \quad \mathbf{R} \in \partial \Omega_{t},
$$

and the static and thermodynamic compatibility conditions on the coherent phase interface

$$
\llbracket \mathbf{P n} \rrbracket=\mathbf{0}, \quad \llbracket \mathbf{n} \cdot \mathbf{b n} \rrbracket=0, \quad \mathbf{R} \in \Gamma,
$$

see $[8,38]$ for details. Hereinafter, we assume coherent phase interface, that is, without discontinuities in displacements on $\Gamma$

$$
\llbracket \mathbf{r} \rrbracket=\mathbf{0}, \quad \mathbf{R} \in \Gamma .
$$

Here, $\mathbf{P}=\frac{\partial W}{\partial \mathbf{F}}$ is the first Piola-Kirchhoff stress tensor, $\mathbf{n}$ is the vector of unit outward normal to $\partial \Omega_{t}$. In addition, the double square brackets in (4) and (5) denote the jump of discontinuity across $\Gamma$ of the corresponding field, $\llbracket(\ldots) \rrbracket=(\ldots)_{+}-(\ldots)_{-}$at $\Gamma$.

In $(4)_{2}, \mathbf{b}$ is the Eshelby tensor defined as follows:

$$
\mathbf{b}=W \mathbf{I}-\mathbf{F}^{T} \mathbf{P},
$$

where I is the unit tensor and the superscript " $T$ " stands for the transposed tensors. For homogeneous material, we have the conservation law, see, e.g. [47, p. 132],

$$
\operatorname{Div} \mathbf{b}=\mathbf{0},
$$

so for any smooth closed surface $S \subset \Omega$ we get

$$
\int_{S} \text { bn } \mathrm{d} A=\mathbf{0} .
$$

Let us consider quasistatic deformations, that is, when the position vectors depend on time-like parameter $t$

$$
\mathbf{r}=\mathbf{r}(\mathbf{R}, t), \quad \mathbf{X}=\mathbf{X}(t),
$$

but we neglect inertial effects. In this case, it can be shown, see, e.g. [42,64], that the energy rate depends on the quasistatic motion of the phase interface as follows

$$
\dot{\mathcal{E}}=\int_{\Gamma} V_{\Gamma} \llbracket \mathbf{n} \cdot \mathbf{b n} \rrbracket \mathrm{d} A,
$$

where the overdot stands for the derivative with respect to time-like parameter $t$ and $V_{\Gamma}$ is the normal velocity of the phase interface. Equation (9) means that $\llbracket \mathbf{n} \cdot \mathbf{b n} \rrbracket$ plays a role of a driving force for the phase interface motion. So (9) results in a kinetic equation

$$
V_{\Gamma}=-F\left(\left.\llbracket \mathbf{n} \cdot \mathbf{b n} \rrbracket\right|_{\Gamma}\right),
$$

where $F=F(\zeta)$ is a kinetic function such that $F(0)=0, \zeta F(\zeta) \geq 0$, see $[1,2,9,10,48]$ for details. So for quasistatic deformations (10) replaces $(4)_{2}$. Linear form of (10) was used for the analysis of instabilities of two-phase solids in [35,64]. 


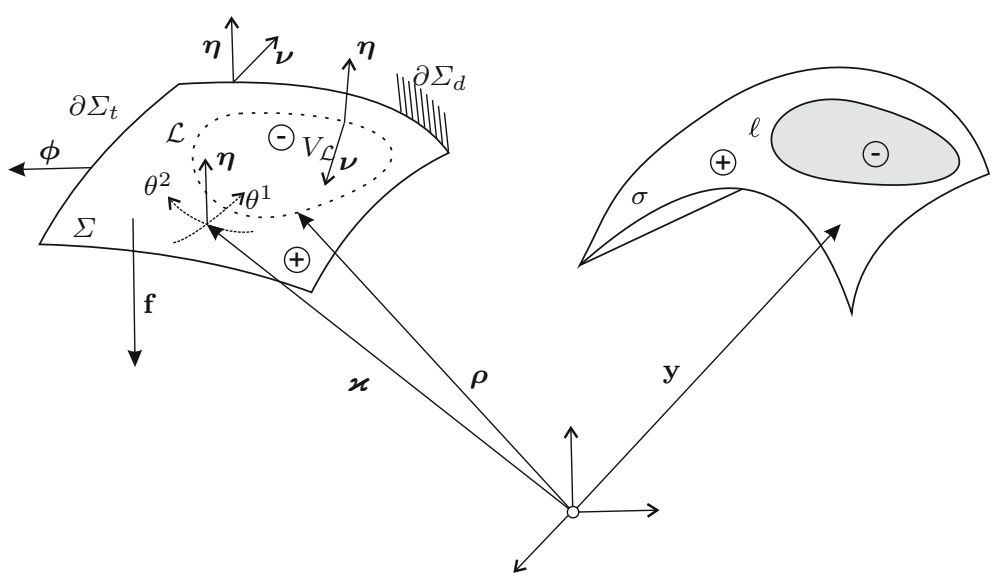

Fig. 2 Deformation of a two-phase shell

\section{Eshelby tensor and equilibrium conditions of two-phase shells}

Following $[16,45,46]$, we consider a shell as a directed material surface which kinematics is described through two kinematically independent surface fields of translations and rotations. In other words, we treat the shell as 2D Cosserat (micropolar) continuum. So in the literature this model is also known as the theory of micropolar shells, see, e.g. $[5,21,23]$ and the reference therein. In the reference placement, the shell is represented by the base surface $\Sigma$ with the position vector $\mathcal{\varkappa}=\boldsymbol{x}\left(\theta^{\alpha}\right)$ and the unit normal vector $\boldsymbol{\eta}=\boldsymbol{\eta}\left(\theta^{\alpha}\right)$, where $\theta^{\alpha}$ are surface curvilinear coordinates, $\alpha=1,2$. In the current placement, the shell is represented by the surface $\sigma$ with the position vector $\mathbf{y}=\mathbf{y}(\boldsymbol{x})$ and the proper orthogonal tensor $\mathbf{Q}=\mathbf{Q}(\boldsymbol{\varkappa})$, as shown in Fig. 2.

For a hyperelastic shell, there exists a surface strain energy function which takes the following form

$$
U=U(\mathbf{E}, \mathbf{K}),
$$

where $\mathbf{E}$ and $\mathbf{K}$ are the referential surface strain measures defined by the formulae

$$
\begin{aligned}
& \mathbf{E}=\mathbf{Q}^{T} \mathbf{F}_{\mathrm{S}}, \quad \mathbf{K}=-\frac{1}{2}\left[\mathbf{Q}^{T} \frac{\partial \mathbf{Q}}{\partial \theta^{\alpha}} \otimes \varkappa^{\alpha}\right]_{\times}, \\
& \mathbf{F}_{\mathrm{S}}=\operatorname{Grad}_{\mathrm{S}} \mathbf{y}, \quad \operatorname{Grad}_{\mathrm{S}}(\ldots)=\frac{\partial}{\partial \theta^{\alpha}}(\ldots) \otimes \boldsymbol{\varkappa}^{\alpha}, \\
& \boldsymbol{x}_{\alpha}=\frac{\partial}{\partial \theta^{\alpha}} \boldsymbol{x}, \quad \boldsymbol{x}_{\alpha} \cdot \boldsymbol{\varkappa}^{\beta}=\delta_{\alpha}^{\beta}, \quad \boldsymbol{\varkappa}^{\alpha} \cdot \boldsymbol{\eta}=0, \quad \alpha, \beta=1,2,
\end{aligned}
$$

where $\mathbf{F}_{\mathrm{S}}$ is the surface deformation gradient and the notation $\mathbf{T}_{\times}$denotes the vectorial invariant of any second-order tensor $\mathbf{T}$, which was introduced by Gibbs [63]. For example, for a diad we have

$$
(\mathbf{a} \otimes \mathbf{b})_{\times}=\mathbf{a} \times \mathbf{b},
$$

where " $x$ " is the cross-product, see for details original lectures by Gibbs in [63, p. 275], and more recent books $[22,43]$. With the vectorial invariant, one can find the axial vector a corresponding to the skew tensor $\mathbf{S}=\mathbf{I} \times \mathbf{a}=\mathbf{a} \times \mathbf{I}$ with the use of the formula

$$
\mathbf{a} \equiv \operatorname{ax}(\mathbf{S})=-\frac{1}{2}(\mathbf{S})_{\times},
$$

where $\operatorname{ax}(\ldots)$ denotes the axial vector associated with the skew tensor (...). Here, we have used the crossproduct between second-order tensor and vector, see [63, p. 280]. This operation can be extended for tensors of any order, see [63, p. 281] and [22,43,52]. For example, for diads we have

$$
(\mathbf{a} \otimes \mathbf{b}) \times \mathbf{c}=\mathbf{a} \otimes(\mathbf{b} \times \mathbf{c}), \quad(\mathbf{a} \otimes \mathbf{b}) \times(\mathbf{c} \otimes \mathbf{d})=\mathbf{a} \otimes(\mathbf{b} \times \mathbf{c}) \otimes \mathbf{d} .
$$

Note that hereinafter we use the same definition of Lagrangian surface gradient Grads and divergence Divs operators as in $[16,24,40]$. 
Similarly to 3D solids undergoing phase transitions, we consider a shell consisting of two phases separated by a phase interface. In the current placement, the phase interface is represented by a smooth curve $\ell$, as shown in Fig. 2. Using Lagrangian description, we introduce the curve $\mathcal{L} \subset \Sigma$ which image under mapping $\mathbf{y}=\mathbf{y}(\varkappa): \Sigma \rightarrow \sigma$ is $\ell$, so $\mathbf{y}(\mathcal{L})=\ell$. We introduce the position vector $\rho$ of $\mathcal{L}$ as an additional kinematical descriptor related to modelling of phase interface quasistatic motion.

Using the variational approach [24], we consider the stationarity of the total energy functional

$$
\mathcal{E}_{\mathrm{S}}[\mathbf{y}, \boldsymbol{\rho}]=\int_{\Sigma}(U-\mathbf{g} \cdot \mathbf{y}) \mathrm{d} A-\int_{\partial \Sigma_{t}} \boldsymbol{\phi} \cdot \mathbf{y} \mathrm{d} s,
$$

where $\mathbf{g}$ and $\boldsymbol{\phi}$ are the surface and boundary forces, respectively. Here, for simplicity we neglect the action of surface and boundary couples. From the variational equation $\delta \mathcal{E}_{\mathrm{S}}=0$ on admissible fields $\mathbf{y}\left(\left.\delta \mathbf{y}\right|_{\partial \Sigma_{d}}=\mathbf{0}\right)$ and $\rho$, we derive the equilibrium equations

$$
\operatorname{Div}_{\mathrm{S}} \mathbf{T}+\mathbf{g}=\mathbf{0}, \quad \operatorname{Div}_{\mathrm{S}} \mathbf{M}+\operatorname{ax}\left(\mathbf{T F}_{\mathrm{S}}^{T}-\mathbf{F}_{\mathrm{S}} \mathbf{T}^{T}\right)=\mathbf{0}, \quad \boldsymbol{x} \in \Sigma,
$$

the static boundary conditions

$$
\mathbf{T} v=\phi, \quad \mathbf{M v}=\mathbf{0}, \quad \varkappa \in \partial \Sigma_{\mathrm{t}},
$$

where $\mathbf{T}$ and $\mathbf{M}$ are the surface stress resultant and stress couple tensors of the first Piola-Kirchhoff type given by the formulae

$$
\mathbf{T}=\mathbf{Q} \frac{\partial U}{\partial \mathbf{E}}, \quad \mathbf{M}=\mathbf{Q} \frac{\partial U}{\partial \mathbf{K}},
$$

$\boldsymbol{v}$ is the surface unit vector externally normal to $\partial \Sigma$, such that $\boldsymbol{v} \cdot \boldsymbol{\eta}=0$, as shown in Fig. 2. In addition, for coherent in rotations phase interface we get the static and thermodynamic compatibility conditions

$$
\begin{aligned}
\llbracket \mathbf{T} v \rrbracket & =\mathbf{0}, \quad \llbracket \mathbf{M} v \rrbracket=\mathbf{0}, \\
\llbracket v \cdot \mathbf{C} v \rrbracket & =\mathbf{0}, \quad \mathbf{C}=U \mathbf{A}-\mathbf{F}_{\mathrm{S}}^{T} \mathbf{T}-\mathbf{K}^{T} \mathbf{Q}^{T} \mathbf{M} ; \quad x \in \mathcal{L}
\end{aligned}
$$

where $\mathbf{A}=\mathbf{I}-\boldsymbol{\eta} \otimes \boldsymbol{\eta}$ is the 2D unit tensor. Following [24] by a coherent in rotations phase interface, we mean an interface without jumps of translations and rotations. Tensor $\mathbf{C}$ is the 2D Eshelby tensor which is full analogue of $\mathbf{b}$. It can be also represented through the surface strain energy and its derivatives

$$
\mathbf{C}=U \mathbf{A}-\mathbf{E}^{T} \frac{\partial U}{\partial \mathbf{E}}-\mathbf{K}^{T} \frac{\partial U}{\partial \mathbf{K}}
$$

Unlike $\mathbf{b}$ for $\mathbf{C}$, there is not a relation similar to (7), that is, $\operatorname{Div}_{S} \mathbf{C} \neq \mathbf{0}$, in general. Indeed, let us note that (7) is valid for homogeneous solids. But a shell is in some sense inhomogeneous, as its properties may depend on the curvature, see, e.g. discussion on uniformity and inhomogeneity of shells in [19,20]. Some exceptions are discussed in [41].

On the other hand, as in the 3D case for quasistatic deformations we have that

$$
\dot{\mathcal{E}}_{\mathrm{S}}=\int_{\mathcal{L}} V_{\mathcal{L}} \llbracket \boldsymbol{v} \cdot \mathbf{C v} \rrbracket \mathrm{d} s,
$$

see [25,26], where $V_{\mathcal{L}}$ is the normal velocity of $\mathcal{L}$. Here, we assume that $\mathbf{y}$ and $\mathbf{Q}$ depend on time-like parameter $t, \mathbf{y}=\mathbf{y}(\varkappa, t), \mathbf{Q}=\mathbf{Q}(\varkappa, t)$. Equation (18) describes the energy release rate for the quasistatic motion of the phase interface. So $\llbracket \boldsymbol{v} \cdot \mathbf{C} \boldsymbol{v} \rrbracket$ plays a role of a driving force acting on the phase interface. The 2D analogy of kinetic equation takes the form

$$
V_{\mathcal{L}}=-F\left(\left.\llbracket v \cdot \mathbf{C v} \rrbracket\right|_{\mathcal{L}}\right),
$$

see $[25,26]$, where the influence of the form of $F$ on the shell deformations was analysed. 


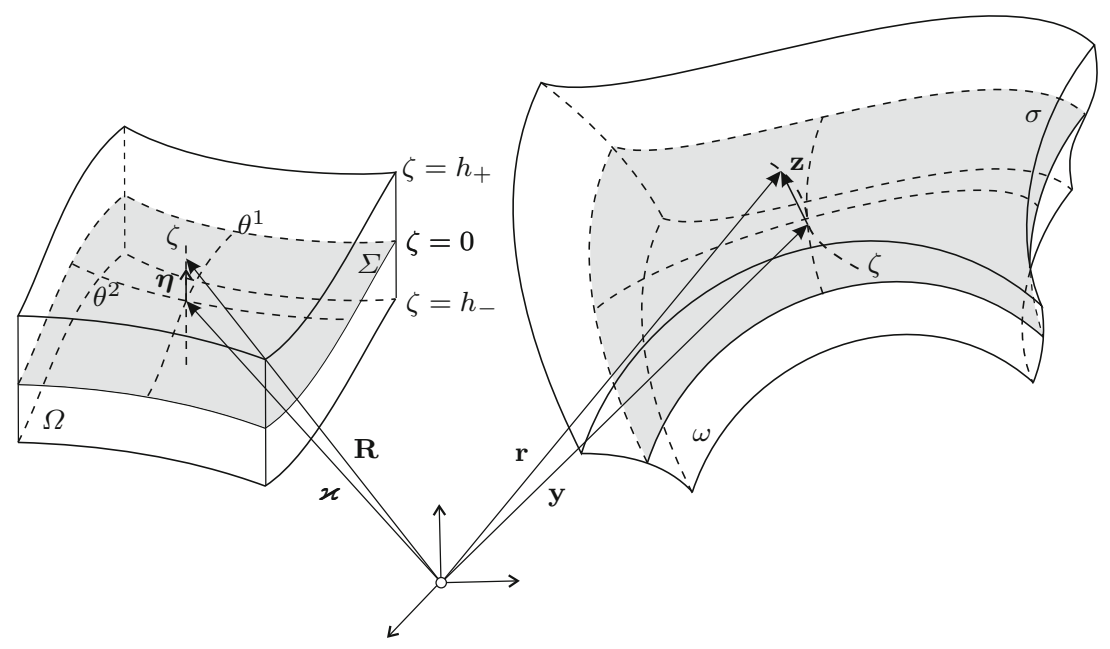

Fig. 3 Deformation of a shell-like body

\section{The through-the-thickness integration procedure}

In previous two sections, we have introduced the Eshelby tensors $\mathbf{b}$ and $\mathbf{C}$ as entirely independent quantities. Indeed, for shells we applied here the so-called direct approach treating a shell as a $2 \mathrm{D}$ elastic continuum $[5,21,23]$. Within the direct approach, one operates with $2 \mathrm{D}$ governing equations without any relation to the 3D nature of a shell, in general. Recently, similar 2D equations were introduced to the new class of thin-walled structures called pantographic lattices, see, e.g. $[6,7,18,31,36]$ and the reference therein. In the literature, various $3 \mathrm{D}$ to $2 \mathrm{D}$ reduction techniques are known, see, e.g. [17,37,46,51] and the recent review [4]. Among these approaches, the through-the-thickness integration of the $3 \mathrm{D}$ equilibrium/motion equations leads to the nonlinear resultant theory of shells. This theory is presented in detail in $[16,45,46,55]$ for finite deformations and in $[22,43]$ for infinitesimal ones. Within this procedure, one derives the $2 \mathrm{D}$ equilibrium/motion equation as the exact consequence of $3 \mathrm{D}$ equations without any kinematical assumptions, whereas $2 \mathrm{D}$ strain measures can be introduced as energetically conjugate to the stress measures.

For example, within the through-the-thickness procedure there is the straightforward correspondence between $W$ and $U$, and between $\mathbf{P}$ and 2D stress measures $\mathbf{T}$ and $\mathbf{M}$. In order to introduce this dependence, let us consider a shell-like solid that is a curved thin-walled body. In the reference placement, this solid occupies a region of the following shape $\Omega=\left\{\left(\theta^{\alpha}, \zeta\right),\left(\theta^{1}, \theta^{2}\right) \in \Sigma,-h_{-} \leq \zeta \leq h_{+}\right.$, where $\Sigma$ is a given referential base surface, and $h=h_{-}+h_{+}$is the shell thickness, as shown in Fig. 3. So in the reference placement the position vector has the following form

$$
\mathbf{R}=\varkappa+\zeta \eta
$$

where $\mathcal{u}$ is the position vector of $\Sigma, \eta$ is the unit normal vector to $\Sigma$, and $\zeta$ is the normal coordinate. In the current configuration, the position vector of the shell is given by

$$
\mathbf{r} \equiv \mathbf{r}(\varkappa, \zeta)=\mathbf{y}(\varkappa)+\mathbf{z}(\varkappa, \zeta)
$$

where $\mathbf{y}$ is the position vector of the shell base surface in the current placement and $\mathbf{z}$ is base reference deviation vector [46].

Let us first consider the strain energy functional for the shell-like body. By the strain energy functional, we mean the total energy functional without external loads. For simplicity, we keep the same notations for these functionals. As $\mathcal{E}$ and $\mathcal{E}_{\mathrm{S}}$ describe the stored energy of the same body, we have to conclude that $\mathcal{E}=\mathcal{E}_{\mathrm{S}}$. So we get that

$$
\mathcal{E} \equiv \int_{\Omega} W \mathrm{~d} \Omega=\int_{\Sigma} U \mathrm{~d} A \equiv \mathcal{E}_{\mathrm{S}} .
$$


As $\mathrm{d} \Omega=\mu \mathrm{d} \zeta \mathrm{d} A$ and

$$
\int_{\Omega}(\ldots) \mathrm{d} \Omega=\int_{\Sigma}\left(\int_{h_{-}}^{h_{+}}(\ldots) \mu \mathrm{d} \zeta\right) \mathrm{d} A
$$

we have

$$
U=\int_{-h_{-}}^{h_{+}} W \mu \mathrm{d} \zeta
$$

where $\mu$ is the geometrical scale factor given by the formula [46]

$$
\mu=1-2 H \zeta+K \zeta^{2}
$$

$H=\frac{1}{2} \operatorname{tr} \mathbf{B}$ and $K=\operatorname{det} \mathbf{B}$ are the mean and Gaussian curvatures of $\Sigma$, respectively, and $\mathbf{B}=-\operatorname{Grad}_{\mathrm{S}} \boldsymbol{\eta}$ is the curvature tensor of $\Sigma$.

Using the identity $[43,46]$

$$
\mathbf{n d} A=(\mathbf{A}-\zeta \mathbf{B})^{-1} \boldsymbol{v} \mu \mathrm{d} \zeta \mathrm{d} s,
$$

we can exactly express the resultant force and the total torque acting on an arbitrary part $A$ of the cross section of the shell-like body

$$
\begin{aligned}
\int_{A} \mathbf{P n d} A= & \int_{\mathcal{L}} \int_{-h_{-}}^{h_{+}} \mathbf{P}(\mathbf{A}-\zeta \mathbf{B})^{-1} \mu \mathrm{d} \zeta \mathrm{d} s \\
\int_{A} \mathbf{r} \times \mathbf{P n d} A= & \int_{\mathcal{L}} \int_{-h_{-}}^{h_{+}}(\mathbf{y}+\mathbf{z}) \times \mathbf{P}(\mathbf{A}-\zeta \mathbf{B})^{-1} \mu \mathrm{d} \zeta \mathrm{d} s \\
= & \int_{\mathcal{L}} \mathbf{y} \times \int_{-h_{-}}^{h_{+}} \mathbf{P}(\mathbf{A}-\zeta \mathbf{B})^{-1} \mu \mathrm{d} \zeta \mathrm{d} s \\
& +\int_{\mathcal{L}}^{h_{-}} \int_{-h_{-}}^{h_{-}} \times \mathbf{P}(\mathbf{A}-\zeta \mathbf{B})^{-1} \mu \mathrm{d} \zeta \mathrm{d} s .
\end{aligned}
$$

In Fig. 4, $A$ is an arbitrary part of the shell-like body cross section with the normal $\mathbf{n}, \mathcal{L}$ is part of the contour of $\Sigma, \mathcal{L}=\partial \Sigma \cap A, s$ and $\tau$ are the arc length parameter and unit tangent vector to $\mathcal{L}$, respectively. Obviously, $A$ is a ruled surface with the parametrization $\mathbf{X}=\mathbf{X}(s, \zeta)=\boldsymbol{\rho}(s)+\zeta \boldsymbol{\eta}(s)$, where $\boldsymbol{\rho}$ is the position vector of $\mathcal{L}$.

Following $[43,46]$, we introduce the stress resultant and the surface couple stress tensors

$$
\mathbf{T}=\int_{-h_{-}}^{h_{+}} \mathbf{P}(\mathbf{A}-\zeta \mathbf{B})^{-1} \mu \mathrm{d} \zeta, \quad \mathbf{M}=\int_{-h_{-}}^{h_{+}} \mathbf{z} \times \mathbf{P}(\mathbf{A}-\zeta \mathbf{B})^{-1} \mu \mathrm{d} \zeta .
$$

With these definitions, (22) and (23) transform into

$$
\int_{A} \operatorname{Pnd} A=\int_{\mathcal{L}} \operatorname{Tv} \mathrm{d} s
$$




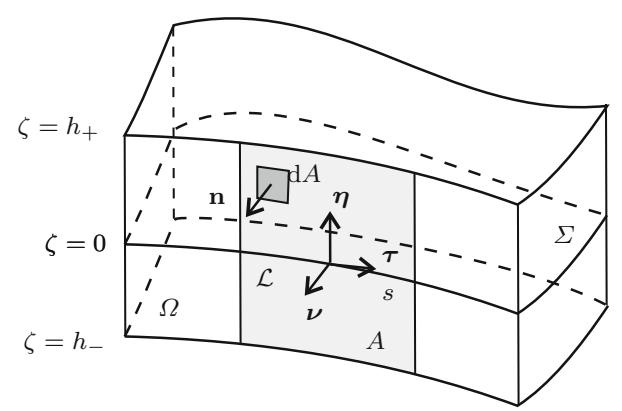

Fig. 4 A part of a shell-like body cross section

$$
\int_{A} \mathbf{r} \times \mathbf{P n d} A=\int_{\mathcal{L}} \mathbf{y} \times \mathbf{T} \boldsymbol{v} \mathrm{d} s+\int_{\mathcal{L}} \mathbf{M} \boldsymbol{v} \mathrm{d} s .
$$

Equations (21) and (24) give the straightforward exact correspondence between defined in the bulk the strain energy $W$ and stress tensor $\mathbf{P}$ and the defined on the base surface function $U$ and strain measures $\mathbf{T}$ and $\mathbf{M}$.

The first correspondence between $\mathbf{b}$ and $\mathbf{C}$ follows from (20). Using (9) and (18), we get the following integral correspondence

$$
\int_{\Gamma} V_{\Gamma} \llbracket \mathbf{n} \cdot \mathbf{b n} \rrbracket \mathrm{d} A=\int_{\mathcal{L}} V_{\mathcal{L}} \llbracket \boldsymbol{v} \cdot \mathbf{C v} \rrbracket \mathrm{d} s .
$$

For such cross section as shown in Fig. 4, we have that

$$
\mathbf{n}=\frac{(\boldsymbol{\tau}-\zeta \mathbf{B} \boldsymbol{\tau}) \times \boldsymbol{\eta}}{|\boldsymbol{\tau}-\zeta \mathbf{B} \boldsymbol{\tau}|}
$$

where $\boldsymbol{\tau}$ is the unit tangent vector to $\mathcal{L}$. From this, it follows that $\mathbf{n} \cdot \boldsymbol{\eta}=0,\left.\mathbf{n}\right|_{\zeta=0}=\boldsymbol{v}$, and that $\left.V_{\Gamma}\right|_{\zeta=0}=$ $V_{\mathrm{L}}=V$. In other words, this means that the velocity of the 2D and 1D phase interfaces coincides with each other in this case. As $V=V(s)$ is an arbitrary function from (27), we get

$$
\llbracket \boldsymbol{v} \cdot \mathbf{C v} \rrbracket=\int_{-h_{-}}^{h_{+}} \llbracket \mathbf{n} \cdot \mathbf{b n} \rrbracket \mu \mathrm{d} \zeta .
$$

This means that integrating through the thickness along normal direction we find the correspondence between $\mathbf{C}$ and $\mathbf{b}$ which follows form the energy correspondence.

To consider this correspondence in detail, let us assume the plane geometry in the reference placement, that is, the case when $\mathbf{B}=\mathbf{0}$ and $\mu=1, \mathbf{n}=\boldsymbol{v}, \boldsymbol{\eta}=$ const. Here, we have that

$$
\operatorname{Grad}(\ldots)=\operatorname{Grad}_{S}(\ldots)+\frac{\partial}{\partial \zeta}(\ldots) \otimes \eta .
$$

So we get that

$$
\mathbf{F}=\operatorname{Grad}_{\mathrm{S}} \mathbf{y}+\operatorname{Grad} \mathbf{z}=\mathbf{F}_{\mathrm{S}}+\operatorname{Grad}_{\mathrm{S}} \mathbf{z}+\frac{\partial \mathbf{z}}{\partial \zeta} \otimes \boldsymbol{\eta} .
$$

Following [16], we represent $\mathbf{z}$ as follows $\mathbf{z}=\mathbf{Q Z}, \quad \mathbf{Z}=\zeta \boldsymbol{\eta}$. This representation corresponds to rigid body rotations of the cross section. More general form of $\mathbf{Z}$ was considered in [16,46]. We get the following identities

$$
\operatorname{Grad}_{\mathrm{S}} \mathbf{z}=\mathbf{Q} \boldsymbol{\eta} \otimes \boldsymbol{\eta}-\zeta \mathbf{Q}(\boldsymbol{\eta} \times \mathbf{K}), \quad\left(\operatorname{Grad}_{\mathrm{S}} \mathbf{z}\right)^{T}=\eta \otimes \mathbf{Q} \boldsymbol{\eta}+\zeta\left(\mathbf{K}^{T} \times \boldsymbol{\eta}\right) \mathbf{Q}^{T}
$$

With these formulae, $\mathbf{b}$ takes the form

$$
\mathbf{b}=W \mathbf{I}-\mathbf{F}_{\mathrm{S}}^{T} \mathbf{P}-(\boldsymbol{\eta} \otimes \mathbf{Q} \eta) \mathbf{P}-\zeta\left(\mathbf{K}^{T} \times \eta\right) \mathbf{Q}^{T} \mathbf{P}
$$


Using the identity

$$
\zeta\left(\mathbf{K}^{T} \times \eta\right) \mathbf{Q}^{T} \mathbf{P}=\mathbf{K}^{T} \mathbf{Q}^{T}(\mathbf{z} \times \mathbf{P})
$$

we transform (29) into

$$
\mathbf{b}=W \mathbf{I}-\mathbf{F}_{\mathrm{S}}^{T} \mathbf{P}-(\boldsymbol{\eta} \otimes \mathbf{Q} \eta) \mathbf{P}-\mathbf{K}^{T} \mathbf{Q}^{T}(\mathbf{z} \times \mathbf{P}) .
$$

Multiplying (30) twice with $\mathbf{n}$, we get

$$
\mathbf{n} \cdot \mathbf{b n}=W-\mathbf{n} \cdot \mathbf{F}_{\mathrm{S}}^{T} \mathbf{P n}-\mathbf{n} \cdot \mathbf{K}^{T} \mathbf{Q}^{T}(\mathbf{z} \times \mathbf{P}) \mathbf{n} .
$$

Finally, integrating this relation through the thickness and taking into account that

$$
\mathbf{T}=\int_{-h_{-}}^{h_{+}} \mathbf{P A} \mathrm{d} \zeta, \quad \mathbf{M}=\int_{-h_{-}}^{h_{+}} \mathbf{z} \times \mathbf{P A} \mathrm{d} \zeta
$$

we obtain that

$$
\begin{aligned}
\int_{-h_{-}}^{h_{+}} \mathbf{n} \cdot \mathbf{b n d} \zeta= & \int_{-h_{-}}^{h_{+}}\left[W-\mathbf{n} \cdot \mathbf{F}_{\mathrm{S}}^{T} \mathbf{P} \mathbf{n}-\mathbf{n} \cdot \mathbf{K}^{T} \mathbf{Q}^{T}(\mathbf{z} \times \mathbf{P}) \mathbf{n}\right] \mathrm{d} \zeta \\
= & \int_{-h_{-}}^{h_{+}} W \mathrm{~d} \zeta-\boldsymbol{v} \cdot \mathbf{F}_{\mathrm{S}}^{T}\left(\int_{-h_{-}}^{h_{+}} \mathbf{P} \mathrm{d} \zeta\right) \boldsymbol{v} \\
& -\boldsymbol{v} \cdot \mathbf{K}^{T} \mathbf{Q}^{T}\left(\int_{-h_{-}}^{h_{+}} \mathbf{z} \times \mathbf{P} \mathrm{d} \zeta\right) \boldsymbol{v} \\
= & U-\boldsymbol{v} \cdot \mathbf{F}_{\mathrm{S}}^{T} \mathbf{T} \boldsymbol{v}-\boldsymbol{v} \cdot \mathbf{K}^{T} \mathbf{Q}^{T} \mathbf{M} \boldsymbol{v} \equiv \boldsymbol{v} \cdot \mathbf{C} \boldsymbol{v} .
\end{aligned}
$$

This relation coincides with the first one given by (28). The case of curved surface $\Sigma$ can be considered similarly, but it requires more lengthy calculations.

\section{Conclusions}

Here, we extended the nonlinear resultant theory of shells to the case of two-phase shells, that is, to the case of thin-walled structures undergoing stress-induced phase transitions. In addition to known 3D to 2D correspondence between the 3D and 2D strain energies $W$ and $U$, between the stress tensor $\mathbf{P}$ and stress resultant $\mathbf{T}$ and surface couple stress $\mathbf{M}$ tensors, we established new straightforward correspondence between 3D and 2D Eshelby tensors. We show that the 2D Eshelby tensor $\mathbf{C}$ can be obtained from its 3D counterpart b using the through-the-thickness integration procedure.

Let us note that in the resultant nonlinear shell theory the reduction in the energy to the 2D requires a special attention. Following [16,46], it can be shown that 2D strain energy can be derived from its 3D counterpart only as a certain approximation. So (21) and (24) are exact dependencies of 2D strain energy and stress resultant and surface couple stress tensors on their 3D counterparts. Nevertheless, the 2D form of $U$ given by (11) is some direct resultant approximation of the 3D strain energy expressed by only $2 \mathrm{D}$ fields defined on $\Sigma$. The same comment should be done for the Eshelby tensors. In other words, the presented correspondence between $\mathbf{C}$ and b has similar approximate nature. Moreover, for the derivation of this correspondence we assume that the phase interface has a form of the normal cross section, that is, also an assumption. For many cases of thin-walled films made of shape memory alloys, such assumption can be validated, see, e.g. experimental observations in $[12,13,30,44,50]$, even for complex microstructure of the interface [11,61]. On the other hand, this assumption is not valid for some experimental observation given in $[59,60]$, where the phase interface is almost parallel to the shell base surface. In such cases, further analysis is required. 
Another field of application of this correspondence is the theory of fracture of thin-walled structures with the use of 2D analogues of energy release rate relations such as discussed in $[41,47,48]$ and path-independent integrals for plates and shells of specific geometry like cylindrical, spherical, etc., see [14,41,54] for the use of the latter within the plate and shell theories. For example, using 2D formulations based on 2D Eshelby tensor one can perform rapid assessments of the stress concentration for thin-walled structures.

Acknowledgements Authors are grateful to Prof. Arkadi Berezovski for the fruitful discussions.

V. Konopińska-Zmysłowska acknowledges the support from the National Centre of Science of Poland with the grant DEC2012/05/D/ST8/02298.

Open Access This article is distributed under the terms of the Creative Commons Attribution 4.0 International License (http:// creativecommons.org/licenses/by/4.0/), which permits unrestricted use, distribution, and reproduction in any medium, provided you give appropriate credit to the original author(s) and the source, provide a link to the Creative Commons license, and indicate if changes were made.

\section{References}

1. Abeyaratne, R., Knowles, J.K.: Kinetic relations and the propagation of phase boundaries in solids. Arch. Ration. Mech. Anal. 114(2), 119-154 (1991)

2. Abeyaratne, R., Knowles, J.K.: Evolution of Phase Transitions. A Continuum Theory. Cambridge University Press, Cambridge (2006)

3. Agrawal, A., Steigmann, D.J.: Coexistent fluid-phase equilibria in biomembranes with bending elasticity. J. Elast. 93(1), 63-80 (2008)

4. Altenbach, H., Eremeyev, V.: Thin-walled structural elements: classification, classical and advanced theories, new applications. In: Altenbach, H., Eremeyev, V. (eds.) Shell-like Structures: Advanced Theories and Applications, pp. 1-62. Springer, Cham (2017)

5. Altenbach, J., Altenbach, H., Eremeyev, V.A.: On generalized Cosserat-type theories of plates and shells: a short review and bibliography. Arch. Appl. Mech. 80(1), 73-92 (2010)

6. Andreaus, U., Spagnuolo, M., Lekszycki, T., Eugster, S.R.: A Ritz approach for the static analysis of planar pantographic structures modeled with nonlinear Euler-Bernoulli beams. Contin. Mech. Thermodyn. 30(5), 1-21 (2018)

7. Barchiesi, E., Ganzosch, G., Liebold, C., Placidi, L., Grygoruk, R., Müller, W.H.: Out-of-plane buckling of pantographic fabrics in displacement-controlled shear tests: experimental results and model validation. Contin. Mech. Thermodyn., pp. $1-13(2018)$

8. Berdichevsky, V.L.: Variational Principles of Continuum Mechanics. I. Fundamentals. Springer, Heidelberg (2009)

9. Berezovski, A., Engelbrecht, J., Maugin, G.A.: Numerical Simulation of Waves and Fronts in Inhomogeneous Solids. World Scientific, New Jersey (2008)

10. Berezovski, A., Maugin, G.A.: On the velocity of a moving phase boundary in solids. Acta Mech. 179(3-4), 187-196 (2005)

11. Bhattacharya, K.: Microstructure of Martensite: Why It Forms and How It Gives Rise to the Shape-Memory Effect?. Oxford University Press, Oxford (2003)

12. Bhattacharya, K., James, R.D.: A theory of thin films of martensitic materials with applications to microactuators. J. Mech. Phys. Solids 47(3), 531-576 (1999)

13. Bhattacharya, K., James, R.D.: The material is the machine. Science 307(5706), 53-54 (2005)

14. Bose, D.K., Kienzler, R.: On material conservation laws for a consistent plate theory. Arch. Appl. Mech. 75(10-12), 607-617 (2006)

15. Boulbitch, A.A.: Equations of heterophase equilibrium of a biomembrane. Arch. Appl. Mech. 69(2), 83-93 (1999)

16. Chróścielewski, J., Makowski, J., Pietraszkiewicz, W.: Statyka i Dynamika Powłok Wielopłatowych. Nieliniowa Teoria i Metoda Elementów Skończonych. Wydawnictwo IPPT PAN, Warszawa (2004)

17. Ciarlet, P.G.: Mathematical elasticity:theory of shells, 2018. North-Holland, Amsterdam (2000)

18. De Angelo, M., Barchiesi, E., Giorgio, I., Abali, B.E.: Numerical identification of constitutive parameters in reduced-order bi-dimensional models for pantographic structures: application to out-of-plane buckling. Arch. Appl. Mech., pp. 1-26 (2019)

19. Epstein, M., De León, M.: On uniformity of shells. Int. J. Solids Struct. 35(17), 2173-2182 (1998)

20. Epstein, M., Roychowdhury, A.: On the notion of embedded homogeneity of thin structures. Math. Mech. Solids 21(6), 657-666 (2016)

21. Eremeyev, V., Altenbach, H.: Basics of mechanics of micropolar shells. In: Altenbach, H., Eremeyev, V. (eds.) Shell-like Structures: Advanced Theories and Applications, pp. 63-111. Springer, Cham (2017)

22. Eremeyev, V.A., Cloud, M.J., Lebedev, L.P.: Applications of Tensor Analysis in Continuum Mechanics. World Scientific, New Jersey (2018)

23. Eremeyev, V.A., Lebedev, L.P., Altenbach, H.: Foundations of Micropolar Mechanics. Springer, Heidelberg (2013)

24. Eremeyev, V.A., Pietraszkiewicz, W.: The non-linear theory of elastic shells with phase transitions. J. Elast. 74(1), 67-86 (2004)

25. Eremeyev, V.A., Pietraszkiewicz, W.: Phase transitions in thermoelastic and thermoviscoelastic shells. Arch. Mech. 61(1), 41-67 (2009)

26. Eremeyev, V.A., Pietraszkiewicz, W.: Thermomechanics of shells undergoing phase transition. J. Mech. Phys. Solids 59(7), 1395-1412 (2011)

27. Eshelby, J.D.: The force on an elastic singularity. Phil. Trans. R. Soc. Lond. A 244(877), 87-112 (1951) 
28. Eshelby, J.D.: The determination of the elastic field of an ellipsoidal inclusion, and related problems. Proc. R. Soc. Lond. Ser. A Math. Phys. Sci. 241(1226), 376-396 (1957)

29. Eshelby, J.D.: The elastic energy-momentum tensor. J. Elast. 5(3-4), 321-335 (1975)

30. Feng, P., Sun, Q.P.: Experimental investigation on macroscopic domain formation and evolution in polycrystalline NiTi microtubing under mechanical force. J. Mech. Phys. Solids 54(8), 1568-1603 (2006)

31. Franciosi, P., Spagnuolo, M., Salman, O.U.: Mean Green operators of deformable fiber networks embedded in a compliant matrix and property estimates. Contin. Mech. Thermodyn., pp. 1-32 (2018)

32. Freidin, A.B.: On the chemical affinity tensor for chemical reactions in deformable materials. Mech. Solids 50(3), 260-285 (2015)

33. Freidin, A.B., Fu, Y.B., Sharipova, L.L., Vilchevskaya, E.N.: Spherically symmetric two-phase deformations and phase transition zones. Int. J. Solids Struct. 43(14-15), 4484-4508 (2006)

34. Freidin, A.B., Vilchevskaya, E.N., Korolev, I.K.: Stress-assist chemical reactions front propagation in deformable solids. Int. J. Eng. Sci. 83, 57-75 (2014)

35. Fu, Y.B., Freidin, A.B.: Characterization and stability of two-phase piecewise-homogeneous deformations. Proc. R. Soc. Lond. A Math. Phys. Eng. Sci. 460(2051), 3065-3094 (2004)

36. Giorgio, I., Harrison, P., dell'Isola, F., Alsayednoor, J., Turco, E.: Wrinkling in engineering fabrics: a comparison between two different comprehensive modelling approaches. Proc. R. Soc. A Math. Phys. Eng. Sci. 474(2216), 20180,063 (2018)

37. Gol'denveizer, A.L.: Theory of Elastic Thin Shells. Pergamon Press, Oxford (1961)

38. Grinfeld, M.: Thermodynamics Methods in the Theory of Heterogeneous Systems. Longman, Harlow (1991)

39. Gurtin, M.E.: Configurational Forces as Basic Concepts of Continuum Physics. Springer, Berlin (2000)

40. Gurtin, M.E., Murdoch, A.I.: A continuum theory of elastic material surfaces. Arch. Ration. Mech. Anal. 57, 291-323 (1975)

41. Kienzler, R., Herrmann, G.: Mechanics in Material Space with Applications to Defect and Fracture Mechanics. Springer, Berlin (2000)

42. Knowles, J.K.: On the dissipation associated with equilibrium shocks in finite elasticity. J. Elast. 9(2), 131-158 (1979)

43. Lebedev, L.P., Cloud, M.J., Eremeyev, V.A.: Tensor Analysis with Applications in Mechanics. World Scientific, New Jersey (2010)

44. Li, Z.Q., Sun, Q.P.: The initiation and growth of macroscopic martensite band in nano-grained NiTi microtube under tension. Int. J. Plast. 18(11), 1481-1498 (2002)

45. Libai, A., Simmonds, J.G.: Nonlinear elastic shell theory. Adv. Appl. Mech. 23, 271-371 (1983)

46. Libai, A., Simmonds, J.G.: The Nonlinear Theory of Elastic Shells, 2nd edn. Cambridge University Press, Cambridge (1998)

47. Maugin, G.A.: Material Inhomogeneities in Elasticity. Chapman Hall, London (1993)

48. Maugin, G.A.: Configurational Forces: Thermomechanics, Physics, Mathematics, and Numerics. CRC Press, Boca Raton (2011)

49. Maugin, G.A., Berezovski, A.: On the propagation of singular surfaces in thermoelasticity. J. Therm. Stress. 32(6-7), 557-592 (2009)

50. Miyazaki, S., Fu, Y.Q., Huang, W.M. (eds.): Thin Film Shape Memory Alloys: Fundamentals and Device Applications. Cambridge University Press, Cambridge (2009)

51. Naghdi, P.: The theory of plates and shells. In: S. Flügge (ed.) Handbuch der Physik, vol. VIa/2, pp. 425-640. Springer, Heidelberg (1972)

52. Naumenko, K., Altenbach, H.: Modeling of Creep for Structural Analysis. Springer, Berlin (2007)

53. Nguyen, T.T., Bruinsma, R.F., Gelbart, W.M.: Elasticity theory and shape transitions of viral shells. Phys. Rev. E 72(5), $051923(2005)$

54. Nicholson, J.W., Simmonds, J.G.: Sanders' energy-release rate integral for arbitrarily loaded shallow shells and its asymptotic evaluation for a cracked cylinder. J. Appl. Mech. 47(2), 363-369 (1980)

55. Pietraszkiewicz, W.: The resultant linear six-field theory of elastic shells: What it brings to the classical linear shell models? ZAMM 96(8), 899-915 (2016)

56. Pietraszkiewicz, W., Eremeyev, V.A., Konopińska, V.: Extended non-linear relations of elastic shells undergoing phase transitions. ZAMM 87(2), 150-159 (2007)

57. Pietraszkiewicz, W., Konopińska, V.: Singular curve in the resultant thermomechanics of shells. Int. J. Eng. Sci. 80, 21-31 (2014)

58. Poluektov, M., Freidin, A.B., Figiel, Ł.: Modelling stress-affected chemical reactions in non-linear viscoelastic solids with application to lithiation reaction in spherical si particles. Int. J. Eng. Sci. 128, 44-62 (2018)

59. Roytburd, A., Slutsker, J.: Coherent phase equilibria in a bending film. Acta Mater. 50(7), 1809-1824 (2002)

60. Roytburd, A.L., Slutsker, J.: Theory of multilayer SMA actuators. Mater. Trans. 43(5), 1023-1029 (2002)

61. Stupkiewicz, S.: Micromechanics of Contact and Interface Layers, Lecture Notes in Applied and Computational Mechanics, vol. 30. Springer, Berlin (2007)

62. Truesdell, C., Noll, W.: The Non-Linear Field Theories of Mechanics. Springer, Berlin (2004)

63. Wilson, E.B.: Vector Analysis, Founded Upon the Lectures of G. W. Gibbs. Yale University Press, New Haven (1901)

64. Yeremeyev, V.A., Freidin, A.B., Sharipova, L.L.: The stability of the equilibrium of two-phase elastic solids. J. Appl. Math. Mech. 71(1), 61-84 (2007)

Publisher's Note Springer Nature remains neutral with regard to jurisdictional claims in published maps and institutional affiliations. 